Preface

\title{
Enhanced Recovery after Surgery (ERAS) for Colorectal Surgery
}

Hiroko Kunitake, MD, MPH ${ }^{1}$

${ }^{1}$ Massachusetts General Hospital, Boston, Massachusetts

Clin Colon Rectal Surg 2019;32:93-94.

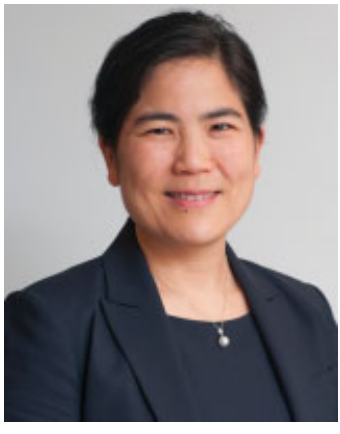

protocols and if ERAS protocols influence patient and surgeon satisfaction. Finally, although ERAS path-

Hiroko Kunitake, MD, MPH modal perioperative care pathways designed to improve the
care and outcomes of patients undergoing surgery. Institutions across the country are adopting ERAS protocols but to varying degrees and with different components included in their protocols making it difficult to determine which components are effective in improving patient care. The focus of this issue of Clinics is to review the components of ERAS protocols and their effectiveness, to illustrate the implementation of an ERAS protocol, and to look at the associated economic implications. In addition, this issue addresses the question of which patients should be excluded from ERAS ways typically are initiated in the immediate preoperative period, the opportunity to extend and enhance the ERAS pathway with prehab is explored. This issue provides a comprehensive evaluation of the current use of ERAS protocols in colorectal surgery to help the practicing colon and rectal surgeon determine which protocol is most effective for them. I would like to thank the contributors for their excellent contributions and Dr. Scott Steele for allowing me to present this information.
Address for correspondence Hiroko Kunitake, MD, MPH, 15 Parkman St., WACC- 460, Boston, MA 02114

(e-mail: hkunitake@partners.org).
Issue Theme Enhanced Recovery after Surgery (ERAS) for

Colorectal Surgery; Guest Editor: Hiroko Kunitake, MD, MPH
Copyright $\odot 2019$ by Thieme Medical Publishers, Inc., 333 Seventh Avenue, New York, NY 10001, USA. Tel: +1(212) 584-4662.
DOI https://doi.org/ 10.1055/s-0038-1676471. ISSN 1531-0043. 